Pacific Journal of Mathematics

ON REGULAR COVERINGS OF 3-MANIFOLDS B 


\title{
ON REGULAR COVERINGS OF 3-MANIFOLDS BY HOMOLOGY 3-SPHERES
}

\author{
E. Luft AND D. SJerve
}

We study homology 3-spheres $\widetilde{M}$ that admit fixed point free actions by a finite group $G$. If $G$ also admits a fixed point free orthogonal action on $S^{3}$ and if certain projective $Z[G]$-modules satisfy a cancellation property we show that the regular covering $\widetilde{M} \rightarrow \widetilde{M} / G$ is induced from a standard regular covering $S^{3} \rightarrow S^{3} / G$ by means of a map $f: \widetilde{M} / G \rightarrow S^{3} / G$ whose degree is relatively prime to the order of $G$ (Theorem 1). We also completely characterize those regular coverings $\widetilde{M} \rightarrow M$ where $M$ is Seifert fibered ( $(4)$. Finally, starting with any given regular covering $\widetilde{M}_{0} \rightarrow M_{0}$ with group of covering transformations $G, M_{0}$ irreducible, and $\widetilde{M}_{0}$ a homology 3-sphere, we show how to construct another regular covering $\widetilde{M} \rightarrow M$ with $\widetilde{M}$ a homology 3-sphere and the same group $G$ of covering transformations, with $M$ sufficiently large, $M$ and $M_{0}$ not homotopy equivalent, and a degree 1 map $f: M \rightarrow M_{0}$ that induces the regular covering $\widetilde{M} \rightarrow M$ from the regular covering $\widetilde{M}_{0} \rightarrow M_{0}$.

1. Introduction. It is a classical result that the finite groups that admit a fixed point free orthogonal action on the 3-sphere $S^{3}$ are exactly the groups of the following four classes (see [ST] or [Mi1]):

(I) The binary polyhedral groups, that is, the binary dihedral groups

$$
Q_{4 n}=\left\{x, y ; x^{2}=(x y)^{2}=y^{n}\right\}, \quad n \geq 2 ;
$$

the binary tetrahedral group

$$
T_{24}=\left\{x, y ; x^{2}=(x y)^{3}=y^{3}, x^{4}=1\right\} ;
$$

the binary octahedral group

$$
O_{48}=\left\{x, y ; x^{2}=(x y)^{3}=y^{4}, x^{4}=1\right\} ;
$$

the binary icosahedral group

$$
I_{120}=\left\{x, y ; x^{2}=(x y)^{3}=y^{5}, x^{4}=1\right\} .
$$

(II) The groups

$$
\begin{aligned}
D\left(2^{k}, 2 l+1\right)=\left\{x, y ; x^{2^{k}}=1, y^{2 l+1}=1, x y x^{-1}=y^{-1}\right\}, \\
k \geq 3, l \geq 1 .
\end{aligned}
$$


(III) The groups

$$
\begin{aligned}
T\left(8,3^{k}\right)=\left\{x, y, z ; x^{2}=\right. & (x y)^{2}=y^{2}, z^{3^{k}}=1, \\
& \left.z x z^{-1}=y, z y z^{-1}=x y\right\}, \quad k \geq 2 .
\end{aligned}
$$

(IV) Cyclic groups $\mathbb{Z}_{m}$ and direct products $\mathbb{Z}_{m} \times G$, where $G$ is any group in classes (I), (II) or (III), with order relatively prime to $m$.

Except for the cyclic groups, the groups $G$ are uniquely determined up to conjugacy in $O(4)$. The orbit manifold $S^{3} / G$ is a spherical space form. If $G$ is not cyclic then $S^{3} / G$ is uniquely determined up to isometry. We refer to the natural covering $q: S^{3} \rightarrow S^{3} / G$ and the action of $G$ on $S^{3}$ as being standard. Each space form $S^{3} / G$ admits a Seifert fibration.

A homology 3-sphere is a 3-manifold with the same homology as a 3-sphere. It was shown in [Mi1] and [L] that if the finite group $G$ acts fixed point freely on some homology 3-sphere, then it must belong to one of the classes (I), .., (IV) or to the following class of groups:

(V) The groups

$$
\begin{aligned}
Q(8 n, k, l)=\left\{x, y, z ; x^{2}=(x y)^{2}=y^{2 n}, z^{k l}\right. & =1, \\
x z x^{-1} & \left.=z^{r}, y z y^{-1}=z^{-1}\right\}
\end{aligned}
$$

where $n, k, l$ are relatively prime odd integers, $r \equiv-1(\bmod k)$ and $r \equiv 1(\bmod l)$ or direct products $\mathbb{Z}_{m} \times Q(8 n, k, l)$ where $m$ and the order $8 n k l$ of the group $Q(8 n, k, l)$ are relatively prime.

Some of the groups in (V) act fixed point freely on some homology 3-spheres and some cannot act fixed point freely on any homology 3sphere (see [DM], p. 278). It is a conjecture that the groups in (V) cannot act fixed point freely on $S^{3}$ (see [Th]).

In this paper we will study those 3-manifolds $M$ which admit regular coverings by a homology 3 -sphere $\widetilde{M}$. Thus if $G$ denotes the group of covering transformations, then $G$ belongs to one of the classes (I), $\ldots,(\mathrm{V})$. If $G$ is in (I), .., (IV) then $G$ has a fixed point free orthogonal action on $S^{3}$. We address the following

Problem. Find conditions under which there is a degree 1 map $f: M \rightarrow S^{3} / G$ so that the covering $p: \widetilde{M} \rightarrow M$ is induced from the standard covering $q: S^{3} \rightarrow S^{3} / G$ by the map $f: M \rightarrow S^{3} / G$.

If $G$ is a cyclic group $\mathbb{Z}_{n}$ then any regular covering $p: \widetilde{M} \rightarrow M$, with $\widetilde{M}$ a homology 3 -sphere and group of covering transformations $\mathbb{Z}_{n}$, can be induced from a standard covering $q: S^{3} \rightarrow S^{3} / \mathbb{Z}_{n}$ by a degree 1 map $f: M \rightarrow S^{3} / \mathbb{Z}_{n}$ onto the lens space $S^{3} / \mathbb{Z}_{n}$ which is determined uniquely up to homotopy equivalence. See [LS2]. 
Suppose $G$ is a finite group. Let $N=\sum_{x \in G} x$ denote the norm element in the integral group ring $Z[G]$. For any integer $r$ the left ideal generated by $r$ and $N$ is denoted by $(r, N)$. If $r$ is relatively prime to the order of $G$, then the ideal $(r, N)$ is a finitely generated projective $Z[G]$-module (see [SW1]). We say that $G$ has the weak cancellation property if $(r, N)$ is free whenever it is stably free. ( $G$ has the cancellation property, if $Z[G] \oplus P \cong Z[G] \oplus Q$ implies that $P \cong Q$ for finitely generated $Z[G]$-modules $P, Q)$.

Amongst the groups in (I), .., (IV) the following are known to have the weak cancellation property:

(1) All cyclic groups. (In fact finite abelian groups have the cancellation property $[\mathrm{SE}]$.)

(2) The groups $T_{24}, O_{48}$ and $I_{120}$.

(3) The groups $Q_{2^{k}}, Q_{4 p}$ with $p$ an odd prime. See [SW2].

The augmentation ideal of $Z[G]$ is denoted by $A[G]$. A $(G, m)$ complex is a finite connected $m$-dimensional CW-complex $X$ with $\pi_{1}(X) \cong G$ and whose universal covering space is $(m-1)$-connected.

In $\S 3$ we prove

THEOREM 1. Let $p: \widetilde{M} \rightarrow M$ be a regular covering of the 3-manifold $M$ by the homology 3-sphere $\widetilde{M}$. Assume that the group $G$ of covering transformations has the weak cancellation property, where $G$ is in one of the classes (I), (II), (III), or (IV). If $q: S^{3} \rightarrow S^{3} / G$ is a standard covering then

(1) The mapping cones $C_{p}$ and $C_{q}$ are homotopy equivalent $(G, 4)$-complexes with $\pi_{4} \cong A[G]$.

(2) There is a map $f: M \rightarrow S^{3} / G$ with degree relatively prime to the order of $G$ and with $f_{*} \pi_{1}(M)=\pi_{1}\left(S^{3} / G\right)=G$ such that the regular covering $p: \widetilde{M} \rightarrow M$ is induced from the standard regular covering $q: S^{3} \rightarrow S^{3} / G$ by the map $f: M \rightarrow S^{3} / G$.

If the manifold $M$ admits a Seifert fibration then either $\widetilde{M}=S^{3}$, $M=S^{3} / G$, and $p: \widetilde{M} \rightarrow M$ is standard, or the group of covering transformations of $p: \widetilde{M} \rightarrow M$ is cyclic and is a transformation group of the Seifert fibration of $\widetilde{M}$ induced by $p: \widetilde{M} \rightarrow M$. See Theorem (4.1). We give explicit descriptions of Seifert fibered homology 3spheres with fixed point free cyclic group actions.

THEOREM 2. Let $p_{0}: \widetilde{M}_{0} \rightarrow M_{0}$ be a regular covering of the irreducible 3-manifold $M_{0}$ by a homology 3-sphere $\widetilde{M}_{0}$ with group of 
covering transformations $G$. Then there is a sufficiently large 3-manifold $M$ containing an incompressible torus, $M$ and $M_{0}$ not homotopy equivalent, a regular covering $p: \widetilde{M} \rightarrow M$ of $M$ by a homology 3-sphere $\widetilde{M}$ with the same group $G$ of covering transformations and a degree 1 map $f: M \rightarrow M_{0}$ such that the regular covering $p: \widetilde{M} \rightarrow M$ is induced from the regular covering $p_{0}: \widetilde{M}_{0} \rightarrow M_{0}$ by $f: M \rightarrow M_{0}$.

Starting with a standard covering $q: S^{3} \rightarrow S^{3} / G$ we can thus construct an abundance of sufficiently large 3-manifolds containing incompressible tori, that admit regular coverings by homology 3-spheres and with group of covering transformations $G$.

Also starting from a fixed point free action of one of the groups $Q(8 n, k, l)$ on some homology 3 -sphere $\widetilde{M}_{0}$ we can thus produce examples of sufficiently large homology 3 -spheres $\widetilde{M}$ containing incompressible tori and admitting fixed point free actions by $Q(8 n, k, l)$.

The case of $G=I_{120}$, the binary icosahedral group (this is the case of nontrivial regular coverings of homology 3-spheres by homology 3-spheres) was considered in [LS1].

2. Preliminaries. Throughout this paper we work in the PL category. A PL homeomorphism we simply call an isomorphism. Our reference for 3-manifold concepts is [He1].

A 3-manifold $M$ is irreducible if each 2-sphere in $M$ bounds a 3 -cell in $M$. Note that if a 3-manifold is regularly covered by a homology 3-sphere, then it is necessarily orientable (the covering transformations must preserve the orientation by the Lefschetz fixed point theorem).

A surface is a connected compact 2-manifold. A surface $F$ in a 3-manifold $M$ is proper if $F \cap \partial M=\partial F$, and it is incompressible in $M$ if it is not a 2-sphere or a 2-cell and if for each 2-cell $D \subset M$ with $D \cap F=\partial D$ there is a 2-cell $D_{0} \subset F$ such that $\partial D_{0}=\partial D$. An orientable connected closed 3-manifold is sufficiently large if it is irreducible and contains a 2 -sided incompressible closed surface.

In [LS2] the following proposition was proved.

Proposition (2.1). Let $W$ be a compact 3-manifold with $\partial W$ a torus. Suppose there is a 1-sphere $S^{1} \subset \partial W$ such that $H_{1}(W)=$ $Z\left[S^{1}\right]$. Then there is a connected proper 2-sided surface $F \subset W$ such that $\partial F$ is a 1-sphere in $\partial W$ and $\partial F$ intersects $S^{1}$ transversally in exactly one point.

The proof of Theorem 1 will be based on the following. 
Proposition (2.2). Suppose $G$ is a finite group with periodic cohomology and with minimal free period $k$. If $G$ has the weak cancellation property then all $(G, M)$-complexes with $m=l k$ and $\pi_{m}(X) \cong$ $A[G]$ as $\pi_{1}$-modules are homotopy equivalent.

Proof. This follows from results in [Dy], see also [LS1]. If $X$ is a $(G, m)$-complex with $\pi_{m}(X) \cong A[G]$, then its algebraic $m$-type is $(G, A[G], r)$ where $r=r(X) \in H^{m+1}\left(G, \pi_{m}(X)\right)=\mathbb{Z}_{|G|}$ is the $k$-invariant, $|G|$ the order of $G$. It is a unit in $\mathbb{Z}_{|G|}$. Therefore $(r, N)$ is a projective ideal. It must be stably free (see Theorem 3.5 of [Dy] - the condition $m \geq 3$ is only needed for one of the directions in this theorem). By hypothesis $(r, N)$ is actually free. According to Corollary (8.4) of [Dy] this means that there is only one isomorphism class of algebraic $m$-types, and therefore only one homotopy type of $(G, m)$-complexes with $\pi_{m}=A[G]$.

3. Proof of Theorem 1. In the following let $G$ be any group from one of the classes (I), (II), (III) or (IV), $p: \widetilde{M} \rightarrow M$ a regular covering with $G$ as group of covering transformations, $\widetilde{M}$ a homology 3-sphere, and $q: S^{3} \rightarrow S^{3} / G$ the regular covering corresponding to any fixed point free orthogonal action of $G$ on $S^{3}$.

If $X$ is a space and $f: X \rightarrow Y$ is a map let $C X, S X$ and $C_{f}$ denote the unreduced cone, suspension, and mapping cone respectively.

Define $W$ to be the space $W=G \times C \widetilde{M} /\left(g_{1}, \tilde{x}, 0\right)=\left(g_{2}, \tilde{x}, 0\right)$. See Figure 1.

Note that $W$ is 3-connected since collapsing one of the cones to a point gives a homotopy equivalence

$$
W=\underbrace{S \widetilde{M} \vee \cdots \vee \widetilde{M}}_{|G|-1 \text { copies }}=\underbrace{S^{4} \vee \cdots \vee S^{4}}_{|G|-1 \text { copies }} .
$$

Also note that there is a natural $G$-action on $W$ defined by $G \times W \dot{\rightarrow}$ $W, h \cdot(g, \tilde{x}, t)=(h g, h(\tilde{x}), t)$ and that

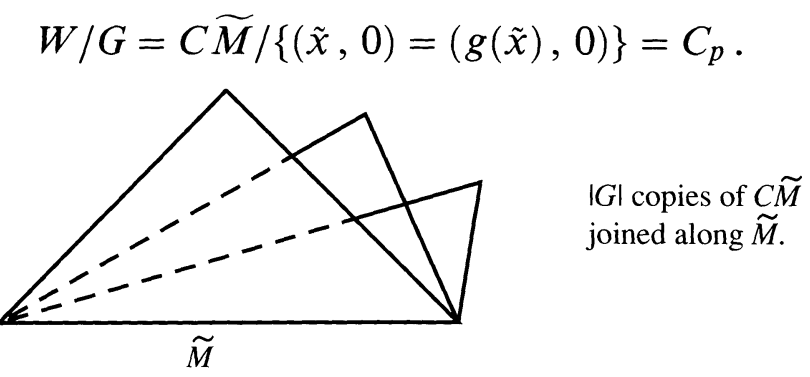

FIGURE 1 
Since this action is fixed point free this implies that $W$ is the universal covering space of $C_{p}$.

Proposition (3.1). $C_{p}$ is a $(G, 4)$-complex with $\pi_{4}\left(C_{p}\right) \cong A[G]$ (as $Z[G]$-modules).

Proof. The same argument as in Lemma 3.3 of [LS1] applies.

Corollary (3.2). Suppose the group $G$ satisfies the weak cancellation property. Then $C_{p}$ and $C_{g}$ are homotopy equivalent.

Proof. This follows immediately from Propositions (3.1) and (2.2). The minimal free period of $G$ is 2 if $G$ is cyclic and nontrivial, and 4 otherwise.

Note. If $L_{n, k}, L_{n, l}$ are lens spaces with fundamental groups $\mathbb{Z}_{n}$, and $p_{k}: S^{3} \rightarrow L_{n, k}, p_{l}: S^{3} \rightarrow L_{n, l}$ are the universal coverings, then $C_{p_{k}}$ and $C_{p_{l}}$ are homotopy equivalent. But $L_{n, k}$ and $L_{n, l}$ are homotopy equivalent if and only if $k l \equiv \pm m^{2}(\bmod n)$ for some $m$ (see e.g. [Co], p. 96).

Proposition (3.3). If $C_{p}$ and $C_{q}$ are homotopy equivalent, then there is a map $f: M \rightarrow S^{3} / G$ so that:

(1) $f_{*} \pi_{1}(M)=\pi_{1}\left(S^{3} / G\right)=G$

(2) $p_{*}\left(\pi_{1}(\widetilde{M})\right)=\operatorname{ker}\left(f_{*}: \pi_{1}(M) \rightarrow G\right)$

(3) the degree of $f$ is relatively prime to the order $|G|$ of $G$.

Proof. Let $h: C_{p} \rightarrow C_{q}$ be a homotopy equivalence and let $i: M \rightarrow$ $C_{p}$ and $j: S^{3} / G \rightarrow C_{q}$ be the inclusions. Note that $C_{q}$ is obtained from $S^{3} / G$ by attaching a 4-cell. By the cellular approximation theorem we therefore can alter $h$ by a homotopy if necessary, so that $h i(M) \subset S^{3} / G$. Let $f=h i: M \rightarrow S^{3} / G$. Thus we have the commutative diagram

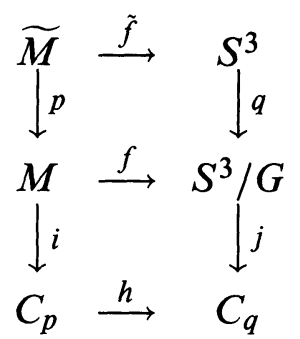

Note that $i_{*}: \pi_{1}(M) \rightarrow \pi_{1}\left(C_{p}\right)$ is an epimorphism and $j_{*}: \pi_{1}\left(S^{3} / G\right)$ $\rightarrow \pi_{1}\left(C_{q}\right)$ is an isomorphism. Therefore $f_{*} \pi_{1}(M)=\pi_{1}\left(S^{3} / G\right)$. 
Since $p_{*} \pi_{1}(\widetilde{M}) \subset \operatorname{ker}\left(f_{*}: \pi_{1}(M) \rightarrow G\right)$, it follows that $p_{*} \pi_{1}(M)=$ $\operatorname{ker}\left(f_{*}: \pi_{1}(M) \rightarrow G\right)$. Property (3) follows from the commutative diagram

$$
\begin{array}{ccc}
H_{3}(M)=Z & \stackrel{f_{*}}{\longrightarrow} & H_{3}\left(S^{3} / G\right) \\
i_{*} \downarrow & & j_{*} \downarrow \\
H_{3}\left(C_{p}\right)=Z_{|G|} & \stackrel{h_{*}}{\longrightarrow} & H_{3}\left(C_{q}\right)=Z_{|G|}
\end{array}
$$

where $i_{*}, j_{*}$ are epimorphisms and $h_{*}$ is an isomorphism.

Corollary (3.2) and Proposition (3.3) prove Theorem 1.

REMARK. It should be noted that a map $f: M \rightarrow S^{3} / G$ inducing the regular covering $p: \widetilde{M} \rightarrow M$ from the covering $q: S^{3} \rightarrow S^{3} / G$ can be constructed by elementary obstruction theory. The properties (1) and (2) are consequences of this construction. The map $f: M \rightarrow S^{3} / G$ and its lift $\tilde{f}: \widetilde{M} \rightarrow S^{3}$ will define by coning a map $h: C_{p} \rightarrow C_{q}$ with $h_{*}: \pi_{1}\left(C_{p}\right) \rightarrow \pi_{1}\left(C_{q}\right)$ an isomorphism. This map, however, will in general not be a homotopy equivalence (which was used to prove property (3)).

The composite $g \cdot f$, where $g: S^{3} / G \rightarrow S^{3} / G$ is any self map inducing an isomorphism on $\pi_{1}$ and having degree relatively prime to $|G|$, will also satisfy the conclusions of Proposition (3.3), and this will change $\operatorname{deg} f$ into the product $(\operatorname{deg} g) \cdot(\operatorname{deg} f)$. We can also alter $\operatorname{deg} f$ to any representative in its congruence class modulo $|G|$. (To see this let $B^{3} \subset M$ be any 3-cell. Then collapsing $\partial B^{3}$ to a point gives a map $c: M \rightarrow M \vee S^{2}$. Consider the composite

$$
M \stackrel{l}{\longrightarrow} M \vee S^{3} \stackrel{f \vee g}{\longrightarrow} S^{3} / G \vee S^{3} / G \stackrel{\nabla}{\longrightarrow} S^{3} / G
$$

where $g: S^{3} \rightarrow S^{3} / G$ has degree $k|G|$ and $\nabla$ is the folding map. Then $\nabla(f \vee g) l$ will satisfy the conclusions of Proposition (3.3), where now the degree is deg $f+k|G|$.)

4. Regular coverings of Seifert fibered 3-manifolds by homology 3spheres. We have the following uniqueness result.

THEOREM (4.1). Let $M$ be a 3-manifold that admits a Seifert fibration, let $p: \widetilde{M} \rightarrow M$ be an non-trivial regular covering by a homology 3-sphere, and let $G$ be the group of covering transformations. Then one of the following holds:

(1) Either $\widetilde{M}=S^{3}, M=S^{3} / G$, and $p: S^{3} \rightarrow S^{3} / G$ is standard.

(2) Or $G$ is cyclic and it is a transformation group of the Seifert 
fibration of $\widetilde{M}$ induced by $p: \widetilde{M} \rightarrow M$ from the Seifert fibration on $M$.

Proof. $\widetilde{M}$ is given the Seifert fibration induced by the regular covering $p: \widetilde{M} \rightarrow M$ from the Seifert fibration on $M$. Then $G$ maps fibers onto fibers.

If $S_{0}^{1} \subset M$ is a regular fiber, then the components of $p^{-1}\left(S_{0}^{1}\right)$ are all regular fibers.

Claim. If $S^{1} \subset M$ is a singular fiber, then either $p^{-1}\left(S^{1}\right)$ is connected, or all components of $p^{-1}\left(S^{1}\right)$ are regular fibers.

Proof of Claim. Suppose $p^{-1}\left(S^{1}\right)$ is not connected and there is a singular fiber $\widetilde{S}^{1} \subset p^{-1}\left(S^{1}\right)$. Since $G$ acts transitively on the components of $p^{-1}\left(S^{1}\right)$ all components of $p^{-1}\left(S^{1}\right)$ are singular fibers and they have the same Seifert invariants. This contradicts the assumption that $\widetilde{M}$ is a homology 3 -sphere (see Satz 12 of [S]).

Case 1. All fibers of $\widetilde{M}$ are regular.

Then by the remark preceding Satz 12 of [S] $\widetilde{M}$ must be the 3sphere. Hence $p: \widetilde{M} \rightarrow M$ is standard.

Case 2. $M$ has a singular fiber $\widetilde{S}^{1}$.

Then $G$ acts without fixed points on $\widetilde{S}^{1}$ and therefore must be cyclic.

It remains to prove that in Case 2, $G$ leaves the fibers setwise fixed. Let $S_{1}, \ldots, S_{s}, S_{s+1}, \ldots, S_{s+t}$ denote the singular fibers in $M$ where $p^{-1}\left(S_{1}\right), \ldots, p^{-1}\left(S_{s}\right)$ are the singular fibers in $\widetilde{M}$ and the components of $p^{-1}\left(S_{S+i}\right), i=1, \ldots, t$, are all regular fibers in $\widetilde{M}$. Let $n_{i}$ be the number of components in $p^{-1}\left(S_{s+i}\right), i=1, \ldots, t$.

The Seifert surface of $\widetilde{M}$ is a 2-sphere (see [S] p. 207). Suppose the Seifert surface of $M$ is a surface with Euler characteristic $2-d$, $d \geq 0$. Let $V_{1}, \ldots, V_{s+t} \subset M$ be disjoint fibered solid tori with centers in the fibers $S_{1}, \ldots, S_{s+t}$. Then $p \mid: \widetilde{M}-p^{-1}\left(V_{1} \cup \cdots \cup V_{s+t}\right) \rightarrow$ $M-\left(V_{1} \cup \cdots \cup V_{s+t}\right)$ is a regular covering projection. The Seifert surface of $M-p^{-1}\left(V_{1} \cup \cdots \cup V_{s+t}\right)$ is a 2-sphere with $s+n_{1}+\cdots+n_{t}$ holes, and the Seifert surface of $M-\left(V_{1} \cup \cdots \cup V_{s+t}\right)$ has $s+t$ holes. The covering projection $p \mid$ induces a covering projection of, the Seifert surfaces. 
Suppose it has $k$ sheets. Then we have the following formula for the Euler characteristics of the Seifert surfaces:

$$
2-\left(s+n_{1}+\cdots+n_{t}\right)=k(2-d-s-t) .
$$

Note that $n_{i} \leq k, i=1, \ldots, t$. Therefore, $(s+d-2) k \leq s-2$. Necessarily, $d=0$. If $s \leq 2, \widetilde{M}$ must be a 3 -sphere and $p: \widetilde{M} \rightarrow M$ is standard.

If $s \geq 3$, then necessarily $k=1$, i.e. the induced covering projection on the Seifert surfaces is an isomorphism. Therefore $G$ leaves each fiber of $\widetilde{M}$ setwise fixed, i.e. $G$ is a transformation group of the Seifert fibration of $M$ (see [S], §14).

A Seifert fibered space $M$ has a unique geometric structure in the sense of Thurston and there are exactly six possible geometries for $M$ determined by the following table (see [Sc1] Theorem 5.3, p. 477):

$$
\begin{array}{cccc} 
& \chi>0 & \chi=0 & \chi<0 \\
e=0 & S^{2} \times \mathbf{R} & E^{3} & H^{2} \times \mathbf{R} \\
e \neq 0 & S^{3} & \text { Nil } & \widetilde{\mathrm{SL}_{2}(\mathbf{R})}
\end{array}
$$

Here $\chi$ is the orbifold Euler characteristic of the Seifert surface $F$, $e$ is the Euler number of the Seifert bundle $M \rightarrow F$, and $S^{2} \times \mathbf{R}$, $S^{3}, E^{3}, \mathrm{Nil}, H^{2} \times \mathbf{R}, \widetilde{\mathrm{SL}_{2}(\mathbf{R})}$ are the six possible universal coverings (geometries) on which $\pi_{1}(M)$ acts by isometries. Note, both $\chi$ and $e$ are rational numbers. If both $M$ and $F$ are orientable then the Seifert invariant of $M$ is $\left(0, o ; g \mid b ; \alpha_{1}, \beta_{1} ; \ldots ; \alpha_{r}, \beta_{r}\right)$, and

$$
\chi=2-2 g-\sum_{i=1}^{r}\left(1-\frac{1}{\alpha_{i}}\right), \quad e=-\left(b+\sum_{i=1}^{r} \frac{\beta_{i}}{\alpha_{i}}\right)
$$

where $g$ is the genus of $F$ and the $\left(\alpha_{i}, \beta_{i}\right), i=1 \cdots r$, are the invariants of the singular fibers. See [SC1] p. 427 and p. 437 respectively.

In $\S 12$ of [S] the following is proved: If $M$ is a homology 3-sphere then $g=0$ and the $\alpha_{1}, \ldots, \alpha_{r}$ are relatively prime in pairs. Moreover, if $M \neq S^{3}$ then $r \geq 3$. Conversely, for any $r \geq 3$ pairwise coprime integers $\alpha_{1}, \ldots, \alpha_{r} \geq 2$ there is a unique Seifert fibered homology 3 -sphere with Seifert invariant $\left(0, o ; o \mid b ; \alpha_{1}, \beta_{1} ; \ldots ; \alpha_{r}, \beta_{r}\right)$. We denote this homology 3 -sphere by $\sum\left(\alpha_{1}, \ldots, \alpha_{r}\right)$.

We have the following proposition ([Mi2], [N]). 
Proposition (4.2). The geometry of a Seifert fibered homology 3sphere is either modelled on $S^{3}$ or on $\widetilde{\mathrm{SL}_{2}(\mathbf{R})}$. The 3-sphere $S^{3}$ and the dodecahedral space $\sum(2,3,5)$ are modelled on $S^{3}$. All other homology 3-spheres $\sum\left(\alpha_{1}, \ldots, \alpha_{r}\right), r \geq 3,\left(\alpha_{1}, \ldots, \alpha_{r}\right) \neq(2,3,5)$ are modelled on $\widehat{\mathrm{SL}_{2}(\mathbf{R}) \text {. }}$

Proof. Let $M$ be a Seifert fibered space with Seifert invariant $(0, o$; $\left.o \mid b ; \alpha_{1}, \beta_{1} ; \ldots ; \alpha_{r}, \beta_{r}\right)$. Then equation (3) in $\S 12$ of [S] states that

$$
b \alpha_{1} \cdots \alpha_{r}+\beta_{1} \alpha_{2} \cdots \alpha_{r}+\alpha_{1} \beta_{2} \alpha_{3} \cdots \alpha_{r}+\cdots+\alpha_{1} \cdots \alpha_{r-1} \beta_{r}+ \pm 1 .
$$

Hence $e= \pm 1 / \alpha_{1} \cdots \alpha_{r} \neq 0$. Consequently, the only possible geometries for $M$ to be modelled on are $S^{3}$, Nil, or $\widetilde{\mathrm{SL}_{2}(\mathbf{R})}$.

Since $g=0, \chi=2-\sum_{i=1}^{r}\left(1-1 / \alpha_{i}\right)$. If $M$ is not $S^{3}$ then $r \geq 3$ and it is easy to show the following

(1) $\chi>0$ only for the unordered triples $(2,2, n),(2,3,3)$, $(2,3,4)$ and $(2,3,5)$

(2) $\chi=0$ only for the unordered triples $(2,3,6),(2,4,4)$, $(3,3,3)$ and for the 4-tuple $(2,2,2,2)$. The only homology 3sphere in this list comes from $(2,3,5)$ since all of the other unordered $r$-tuples $\left(\alpha_{1}, \ldots, \alpha_{r}\right)$ are not relatively prime in pairs.

The homology 3-spheres $\sum\left(\alpha_{1}, \ldots, \alpha_{r}\right), r \geq 3$, have representations as follows (see $[\mathbf{N}])$.

Let $a_{i j} \in \mathbb{C}, i=1, \ldots, r-2, j=1, \ldots, r$, be such that every $(r-2) \times(r-2)$ submatrix of the $(r-2) \times r$ matrix $A=\left(a_{i j}\right)$ is non-singular. Then

$$
\begin{aligned}
& V_{A}\left(\alpha_{1}, \ldots, \alpha_{r}\right) \\
& \quad=\left\{\left(z_{1}, \ldots, z_{r}\right) \in \mathbb{C}^{r}: a_{i 1} z_{1}^{\alpha_{1}}+\cdots+a_{i r} z_{r}^{\alpha_{r}}=0, i=1, \ldots, r-2\right\}
\end{aligned}
$$

is a complex algebraic surface which is non-singular except at 0 . Let $S^{2 r-1}=\left\{\left(z_{1}, \ldots, z_{r}\right) \in \mathbb{C}^{r}:\left|z_{1}\right|^{2}+\cdots+\left|z_{r}\right|^{2}=1\right\}$ be the unit sphere in $\mathbb{C}^{r}$.

Then

$$
\sum\left(\alpha_{1}, \ldots, \alpha_{r}\right)=V_{A}\left(\alpha_{1}, \ldots, \alpha_{r}\right) \cap S^{2 r-1} .
$$

In particular, the diffeomorphism type of $\sum\left(\alpha_{1}, \ldots, \alpha_{r}\right)$ is independent of the matrix $A$.

If $r=3$, we may choose $A=(1,1,1)$ and obtain

$$
\sum\left(\alpha_{1}, \alpha_{2}, \alpha_{3}\right)=\left\{\left(z_{1}, z_{2}, z_{3}\right) \in \mathbb{C}^{3}: z_{1}^{\alpha_{1}}+z_{2}^{\alpha_{2}}+z_{3}^{\alpha_{3}}=0\right\} \cap S^{5} .
$$


Let $G=\mathbb{Z}_{n}$ be a cyclic group and suppose that $n$ is relatively prime to each of $\alpha_{1}, \ldots, \alpha_{r}$. Then $\mathbb{Z}_{n}$ acts on $\sum\left(\alpha_{1}, \ldots, \alpha_{r}\right)$ without fixed points as a transformation group of the Seifert fibration as follows:

$$
\begin{aligned}
& \mathbb{Z}_{n} \times \sum\left(\alpha_{1}, \ldots, \alpha_{r}\right) \quad \longrightarrow \quad \sum\left(\alpha_{1}, \ldots, \alpha_{r}\right) \\
& \left(t^{i},\left(z_{1}, \ldots, z_{r}\right)\right) \longrightarrow\left(\xi^{\alpha / \alpha_{1} i} z_{1}, \ldots, \xi^{\alpha / \alpha_{r} i} z_{r}\right)
\end{aligned}
$$

where $t$ is a generator of $\mathbb{Z}_{n}, \alpha=\alpha_{1} \cdots \alpha_{r}$ and $\xi$ is a primitive $n$th root of unity. We call any conjugate of this action a standard action of $\mathbb{Z}_{n}$ on the homology 3-sphere $\sum\left(\alpha_{1}, \ldots, \alpha_{r}\right)$.

Proposition (4.3). Let $G$ be a group acting fixed point freely on the homology 3-sphere $\sum\left(\alpha_{1}, \ldots, \alpha_{r}\right)$. Then $G=Z_{n}$ and the action is standard.

Proof. $\sum\left(\alpha_{1}, \ldots, \alpha_{r}\right) / G$ has a Seifert fibered structure (see [Sc2] p. 35). By Theorem (4.1), $G=\mathbb{Z}_{n}$ is a transformation group of the Seifert fibration of $\sum\left(\alpha_{1}, \ldots, \alpha_{r}\right)$ induced by the regular covering $p: \sum\left(\alpha_{1}, \ldots, \alpha_{r}\right) \rightarrow \sum\left(\alpha_{1}, \ldots, \alpha_{r}\right) / G$. But the Seifert fibration of $\sum\left(\alpha_{1}, \ldots, \alpha_{r}\right)$ is unique (see [S] Satz 12).

Thus we have the following

Corollary (4.4). Let $M$ be a Seifert fibered 3-manifold and let $p: \widetilde{M} \rightarrow M$ be a regular covering by a homology 3-sphere with cyclic group of covering transformations. Then either $\widetilde{M}=S^{3}$ or $\widetilde{M}=$ $\sum\left(\alpha_{1}, \ldots, \alpha_{r}\right)$ and the action is standard.

5. Proof of Theorem 2. Let $W$ be an irreducible orientable compact 3-manifold with $\partial W$ a torus and with $H_{1}(W)=\mathbb{Z}, W$ not a solid torus. (e.g. let $X$ be any irreducible homology 3-sphere with $\pi_{1}(X) \neq$ 1 and $S^{1} \subset X$ a 1-sphere not nullhomotopic in $X$, or $X=S^{3}$ and $S^{1} \subset S^{3}$ a knot. Then $W=\overline{X-N\left(S^{1}\right)}$, where $N\left(S^{1}\right)$ is a regular neighborhood of $S^{1}$ in $X$, is such an irreducible orientable compact 3-manifold). Note that $\partial W$ is incompressible in $W$. By Proposition (2.1) there is a connected proper 2-sided surface $F \subset W$ such that $\partial F$ is a 1 -sphere in $\partial W$, and $\partial F$ intersects $S^{1}$ transversally in exactly one point. Let $\partial W=S^{1} \times \partial F$ be such that $\left[S^{1}\right]$ is a generator of $H_{1}(W)$.

By a result of [Ha], there is a 1 -sphere $S_{0}^{1} \subset M_{0}$ which is null homotopic in $M_{0}$ and such that $C=\overline{M_{0}-N\left(S_{0}^{1}\right)}$ is a fiber bundle over 
a 1-sphere with fiber a proper surface $F_{0}$, where $N\left(S_{0}^{1}\right)=S_{0}^{1} \times D_{0}^{2}$ is a regular neighborhood of $S_{0}^{1}$ in $M_{0}$. Note that $C$ is irreducible and the torus $\partial C$ is incompressible in $C$. Let $g:(W, \partial W) \rightarrow\left(N\left(S_{0}^{1}\right)\right.$, $\left.\partial N\left(S_{0}^{1}\right)\right)$ be a map such that $g \mid: \partial W \rightarrow \partial N\left(S_{0}^{1}\right)$ is an isomorphism, $g(F)=D_{0}^{2}$ and $g_{*}\left[S^{1}\right]=\left[S_{0}^{1}\right]$ in $H_{1}\left(\partial N\left(S_{0}^{1}\right)\right)$. Define

$$
M=W \cup C / x=g(x), \quad x \in \partial W
$$

and the map $f: M \rightarrow M_{0}$ by

$$
f(x)= \begin{cases}x, & x \in C, \\ g(x), & x \in W .\end{cases}
$$

The closed 3-manifold $M$ is orientable, irreducible and the torus $\partial W=\partial C$ is incompressible in $M$. The map $f: M \rightarrow M_{0}$ has degree 1 .

Let $p: \widetilde{M} \rightarrow M$ be the regular covering induced from the regular covering $p_{0}: \widetilde{M}_{0} \rightarrow M_{0}$ by the degree 1 map $f: M \rightarrow M_{0}$. The same Mayer-Vietoris sequence argument as in the proof of Theorem (5.1) of [LS1] applies to prove that $\widetilde{M}$ is a homology 3-sphere.

Lastly, $M$ and $M_{0}$ cannot be homotopy equivalent since $\pi_{1}(M)$ and $\pi_{1}\left(M_{0}\right)$ cannot be isomorphic. To see this, note that $f_{*}: \pi_{1}(M) \rightarrow$ $\pi_{1}\left(M_{0}\right)$ is an epimorphism with $\operatorname{ker}\left(f_{*}\right) \neq 1$. Thus if $\pi_{1}(M) \cong$ $\pi_{1}\left(M_{0}\right)$, then $\pi_{1}(M) \cong \pi_{1}(M) / \operatorname{ker}\left(f_{*}\right)$ and $\pi_{1}(M)$ is not Hopfian. But $M$ is sufficiently large and therefore $\pi_{1}(M)$ is residually finite and hence Hopfian (see [He2]), a contradiction.

REMARK. Since $M$ is orientable and irreducible it follows from a result of $[\mathbf{D u}]$ that $\widetilde{M}$ is also irreducible.

\section{REFERENCES}

[CO] M. Cohen, $A$ course in simple-homotopy theory, Grad. Texts in Math., Vol. 10 (1973), Springer-Verlag.

[DM] J. Davis and R. Milgram, A survey of the spherical space form problem, Math. Reports, vol. 2 (part 2) (1985), Harwood Academic Publ. GmbH.

[Du] M. Dunwoody, An equivariant sphere theorem, Bull. London Math. Soc., 17 (1985), 437-448.

[Dy] M. Dyer, Homotopy classification of $(\pi, m)$-complexes, J. Pure Appl. Algebra, 7 (1976), 249-282.

[Ha] J. Harer, Representing elements of $\pi_{1}\left(M^{3}\right)$ by fibered knots, Math. Proc. Cambridge Philos. Soc., 92 (1982), 133-138.

[He1] J. Hempel, 3-manifolds, Ann. of Math. Studies, 86 (1976), Princeton Univ. Press.

[He2] __ Residual finiteness for 3-manifolds, Ann. of Math. Studies, 111 (1987), 379-396. 
[L] R. Lee, Semicharacteristic classes, Topology, 12 (1973), 183-199.

[LS1] E. Luft and D. Sjerve, Regular coverings of homology 3-spheres by homology 3-spheres, Trans. Amer. Math. Soc., 311 (1989), 467-481.

[LS2] _ Degree 1 maps into lens spaces and free cyclic actions on homology 3-spheres, Topology and its Applications, 37 (1990), 131-136.

[Mi1] J. Milnor, Groups which act on $S^{n}$ without fixed points, Amer. J. Math., 79 (1957), 623-631.

[Mi2] _ On the 3-dimensional Brieskorn manifolds $M(p, q, r)$, Ann. of Math. Studies, 84 (1975), 175-225.

[N] W. Neumann, Brieskorn complete intersections and automorphic forms, Invent. Math., 42 (1977), 285-293.

[Sc1] P. Scott, The geometries of 3-manifolds, Bull. London Math. Soc., 15 (1983), 401-487.

[Sc2] _ There are no fake Seifert fiber spaces with infinite $\pi_{1}$, Ann. of Math., 117 (1983), 35-70.

[S] H. Seifert, Topologie dreidimensionaler gefaserter Räume, Acta Math., 60 (1933), 147-238. English translation in: M. Seifert and W. Threlfall. A textbook of Topology, Academic Press (1980).

[ST] H. Seifert and W. Threlfall, Topologische Untersuchung der Diskontinuitätsbereiche endlicher Bewegungsgruppen des dreidimensionalen sphärischen Raumes, Math. Annalen, 104 (1930-1931), 1-70.

[Sw1] R. Swan, Induced representations and projective modules, Ann. of Math., 71 (1960), 552-578.

[Sw2] _ Projective modules over binary polyhedral groups, J. Reine Angew. Math., 342 (1983), 66-172.

[SE] R. Swan and E. Evans, $K$-theory of finite groups and orders, Lect. Notes in Math. Vol. 149 (1970), Springer-Verlag.

[Th] C. Thomas, $A$ reduction theorem for free actions by the group $Q(8 n, k, l)$ on $S^{3}$, Bull. London Math. Soc., 20 (1988), 65-67.

Received August 15, 1989 and in revised form February 28, 1991. Research partially supported by NSERC grants A3503 and A7218.

The University of British Columbia

VANCOUVER, BRITISH COLUMBIA

Canada, V6T 1 Y4 



\title{
PACIFIC JOURNAL OF MATHEMATICS EDITORS
}

\author{
V. S. VARADARAJAN \\ (Managing Editor) \\ University of California \\ Los Angeles, CA 90024-1555-05 \\ Herbert Clemens \\ University of Utah \\ Salt Lake City, UT 84112 \\ F. Michael Christ \\ University of California \\ Los Angeles, CA 90024-1555 \\ THOMAS ENRIGHT \\ University of California, San Diego \\ La Jolla, CA 92093
}

Nicholas ERcolani

University of Arizona

Tucson, AZ 85721

R. FINN

Stanford University

Stanford, CA 94305

VAUGHAN F. R. JoNeS

University of California

Berkeley, CA 94720

C. C. MOORE

University of California

Berkeley, CA 94720

MARTIN SchaRLEMANN

University of California

Santa Barbara, CA 93106

HAROLd StaRK

University of California, San Diego

La Jolla, CA 92093

SteVen Kerckhoff

Stanford University

Stanford, CA 94305

\section{ASSOCIATE EDITORS}

\begin{tabular}{|c|c|c|c|c|}
\hline R. ARENS & $\begin{array}{l}\text { E. F. BECKENBACH } \\
(1906-1982)\end{array}$ & NeumanN & $\begin{array}{c}\text { F. WoLF } \\
(1904-1989)\end{array}$ & K. YoshidA \\
\hline \multicolumn{5}{|c|}{ SUPPORTING INSTITUTIONS } \\
\hline \multicolumn{2}{|c|}{ JNIVERSITY OF ARIZONA } & \multicolumn{3}{|c|}{ UNIVERSITY OF OREGON } \\
\hline \multicolumn{2}{|c|}{ UNIVERSITY OF BRITISH COLUMBIA } & \multicolumn{3}{|c|}{ UNIVERSITY OF SOUTHERN C } \\
\hline \multicolumn{2}{|c|}{ CALIFORNIA INSTITUTE OF TECHNOLOGY } & \multirow{2}{*}{\multicolumn{3}{|c|}{ STANFORD UNIVERSITY }} \\
\hline \multicolumn{2}{|c|}{ UNIVERSITY OF CALIFORNIA } & & & UNIVERSITY OF HAWAII \\
\hline \multicolumn{2}{|c|}{ MONTANA STATE UNIVERSITY } & \multicolumn{3}{|c|}{ UNIVERSITY OF TOKYO } \\
\hline \multicolumn{2}{|c|}{ UNIVERSITY OF NEVADA, RENO } & \multicolumn{3}{|c|}{ UNIVERSITY OF UTAH } \\
\hline \multicolumn{2}{|c|}{ NEW MEXICO STATE UNIVERSITY } & \multicolumn{3}{|c|}{ DN STATE UNIVERSITY } \\
\hline \multicolumn{2}{|c|}{ OREGON STATE UNIVERSITY } & UNIVER & OF WASHIN & \\
\hline
\end{tabular}




\section{Pacific Journal of Mathematics}

Vol. 152, No. $1 \quad$ January, 1992

B. V. Rajarama Bhat, On a characterization of velocity maps in the space of observables .......................................

John David Brillhart, Note on the discriminant of certain cyclotomic period

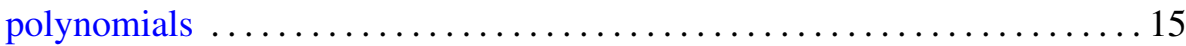

Steven R. Costenoble and Stefan Waner, The equivariant Thom

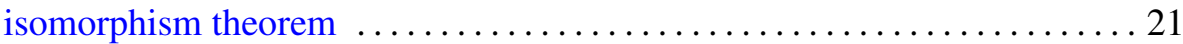

Karl Heinz Dovermann and Dong Youp Suh, Smith equivalence for finite

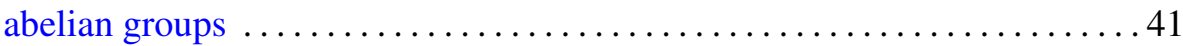

Klaus-Jochen Engel, On singular perturbations of second order Cauchy

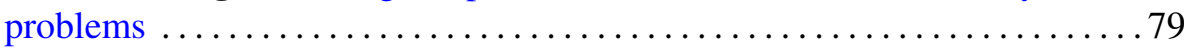

Angel Ferrandez and Pascual Lucas, On surfaces in the 3-dimensional

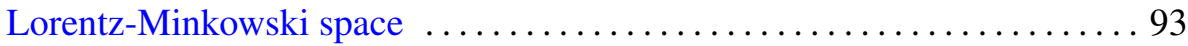

Brian Hartley, A general Brauer-Fowler theorem and centralizers in locally

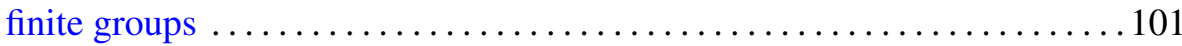

Joel Hass, Intersections of least area surfaces .................... 119

Edward Kissin, Indices of unbounded derivations of $C^{*}$-algebras $\ldots \ldots \ldots 125$

Erhard Luft and Denis Karmen Sjerve, On regular coverings of

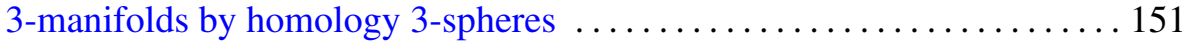

Daniel Pecker, Sur la projection de variétés algébriques réelles .......... 165

Claus Scheiderer, Some remarks on orderings under finite field

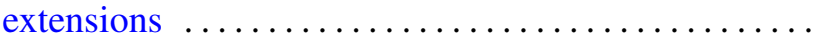

Fernando Serrano, Elliptic surfaces with an ample divisor of genus two 